\title{
Ab Initio Investigations of Iron-Based Martensitic Systems
}

\author{
H.C. Herper, E. Hoffmann and P. Entel \\ Gerhard-Mercator-Universität Duisburg, Theoretische Tieftemperaturphysik, Lotharstrasse 1, \\ 47048 Duisburg, Germany
}

\begin{abstract}
We apply a full-potential first-principles method to investigate the electronic-structure and elastic features of pure iron and ordered transition metal systems like Fe-Ni and Fe-Mn. The martensitic phase transformations in these compounds mainly depend on the properties of iron. Therefore the phase diagram of iron has been studied in detail in view of structural transformations and magnetic order. To this the total energy and magnetic moment have been investigated as function of the atomic volume. In agreement with experimental results, we find a ferromagnetic bcc ground-state and an antiferromagnetic fcc phase having a higher energy of $7 \mathrm{mRy}$. The resulting pressure for the bcc $\rightarrow$ hcp transition is about $11.5 \mathrm{GPa}$ in agreement with experiments. Besides that, total energy calculations have been done for stoichiometric Fe-Mn and Fe-Ni compounds. The resulting alloy phase diagrams are in a good agreement with the experimental data.
\end{abstract}

\section{INTRODUCTION}

In many ferrous transition metal alloys there is a strong interplay of structural and magnetic phase transitions [1]. Earlier theoretical investigations suggested that the martensitic $\gamma \rightarrow \alpha$ transformation in pure iron is driven by the onset of ferromagnetic order [2]. In this paper we try to examine in how far this iron feature is conserved in ferrous transition metal alloys. For a long time it was not possible to get a satisfying theoretical description of iron. Many methods give only a partially correct description of the sequence of energies associated with the different structural and magnetic ground states $[3,4]$. In order to understand better the connection between magnetic order and structural transitions it is useful to study again iron in detail. Therefore, we made use of a full-potential band-structure theory in the density functional formalism together with the generalized gradient approximation (GGA) which provides a proper description of iron. The resulting zero temperature phase diagram compares well with experimental findings. The resulting energy differences between the different phases allow to draw some conclusions about the transition temperatures, thereby reproducing experimental results qualitatively well. Next we extended the calculations to transition metal alloys. In this paper we focus on the Fe-Mn and Fe-Ni systems with $\mathrm{Mn}$ and $\mathrm{Ni}$ having both a close-packed ground state structure but different magnetic behavior, which allows us to check the influence of iron in different magnetic environments. Because of the need of a large computational effort calculations were restricted to the fcc and bcc phases. Though manganese has a more complex structure, we still can extract chemical trends from these restricted calculations and apart from that fcc $\mathrm{Mn}$ is the technologically more interesting material.

\section{METHOD}

The structural and magnetic phase diagrams of the two martensitic systems, $\mathrm{Fe}-\mathrm{Ni}$ and $\mathrm{Fe}-\mathrm{Mn}$, are obtained from full-potential energy calculations for the stoichiometric compounds $\mathrm{Fe}_{\mathrm{e}} \mathrm{Fe}_{3} \mathrm{X}, \mathrm{FeX}, \mathrm{X}$ $[\mathrm{X}=\mathrm{Ni}, \mathrm{Mn}]$. We have studied the ferromagnetic (FM), nonmagnetic (NM) and antiferromagnetic 
(AFM) states of these systems. In the present work the AFM state is always a layered AF1 structure, see Fig. 1. This case requires only a relatively small unit cell (4 atoms per cell) compared to other antiferromagnetic orderings.

The lattice constants, total energies and magnetic moments have been calculated within the full

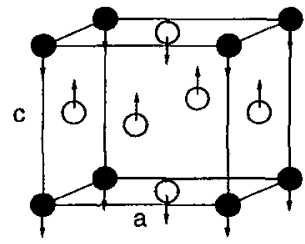

Figure 1: Representation of the tetragonal unit cell (fcc: $c / a=1.0$, bcc: $c / a=1 / \sqrt{2}$ ). Black and white circles mark (different) sorts of atoms. The spin order corresponds to a layered antiferromagnet.

potential linearized augmented plane-wave (FLAPW) method employing the WIEN95 code [6]. A detailed description of the method can be find in Ref. [5]. All investigations were performed using the generalized gradient approximation (GGA II) for the exchange and correlation potential established by Perdew and Wang [7]. This is unavoidable for iron rich systems as well as for iron itself, otherwise total energy calculations would produce a hexagonal ground state of iron [3].

Static ground state properties like equilibrium volume and bulk modulus $B_{0}$ were extracted from total energy calculations using fits to the Murnaghan's equation of state [8]. For more computational details see $[9,10]$.

\section{RESULTS}

\subsection{Ground state properties of $\mathrm{Mn}, \mathrm{Fe}$ and $\mathrm{Ni}$}

We start by discussing the pure transition metals. The calculated equilibrium volume $V_{0}$, saturation magnetization $M_{0}$ and bulk moduli $B_{0}$ for the bcc and fcc ground states are given in Table 1 together with the experimental values. The calculated equilibrium volumes are always smaller than the experimental ones. The maximum deviation is obtained for antiferromagnetic fcc Mn (about 7\%) and fcc Fe (5.4\%). This can be understood from two facts. First, we used ideal fcc and bcc lattices instead of tetragonal distorted lattices. Second, we did not take into account zero point lattice energies. For manganese there is an additional problem because the experimental equilibrium volume has been extrapolated from data corresponding to $\mathrm{Cu}-\mathrm{Mn}$, Ni-Mn and Fe-Mn alloys.

The magnetic moments of all investigated phases are in good agreement with experimental findings. The largest discrepancy occurs for AFM $\gamma$-Fe, however the value cited in Table 1 is only a lower limit for the magnetic moment of AFM fcc Fe [15]. Summarizing we can say that the ground state properties of $\mathrm{Mn}, \mathrm{Fe}$ and $\mathrm{Ni}$ are well reproduced.

In the following we will discuss the iron phase diagram in detail (see Fig.1) baring in mind that the martensitic phase transformations of $\mathrm{Fe}-\mathrm{Ni}$ and $\mathrm{Fe}-\mathrm{Mn}$ occur in the iron rich region. In agreement with experiment we found a ferromagnetic bcc ground state. The lowest lying fcc phase has antiferromagnetic order and is about $7.1 \mathrm{mRy}$ higher in energy than the FM bcc state. This energy difference can be related to the experimental $\alpha \rightarrow \gamma$ transition temperature $T_{0}$. Furthermore a nonmagnetic hep state exists $6 \mathrm{mRy}$ above the bcc ground state having an equilibrium volume of $V_{0}=69.94 \mathrm{a} . \mathrm{u}$. The calculated transition pressure amounts to $11.5 \mathrm{GPa}$ which is comparable to the experimental value of $13.6 \mathrm{GPa}$. The ferromagnetic fcc phase has two local minima, a low-moment low-spin state (LS) and a high-moment high-spin (HS) state. Especially the HS state is important for the discussion of magnetovolume instabilities in iron. From this the Anti-Invar effect can be understood as a transition from AFM $\gamma$-Fe to FM HS $\gamma$-Fe caused by thermal excitations. This is connected to a volume expansion of about $12.5 \%$ compared to the experimentally observed value of 
Table1: Calculated atomic volume $\left(V_{0}\right)$, bulk modulus $\left(B_{0}\right)$ and magnetic moment $\left(M_{0}\right)$ for the ground states of the fcc and bcc structure. Additionally the hcp data of iron are presented. Experimental data are given in brackets. All theoretical results are calculated with the FLAPW method using GGA.

\begin{tabular}{clrlll}
\hline \hline System & \multicolumn{1}{l}{ Structure } & $V_{0} /$ atom(a.u. $\left.{ }^{3}\right)$ & $B_{0}(M b a r)$ & $\mathrm{M}\left(\mu_{B}\right)$ \\
\hline $\mathrm{Mn}$ & bcc & FM & 73.98 & 2.53 & $1.00(1.00)^{a}$ \\
& fcc & AFM & $76.79(82.70)^{b}$ & 1.51 & $1.90(2.40)^{a}$ \\
$\mathrm{Fe}$ & bcc & FM & $76.95(78.94)^{c}$ & $1.86(1.68)^{d}$ & $2.17(2.22)^{d}$ \\
& fcc & AFM & $72.16(76.24)^{c}$ & 1.93 & $1.30(0.75)^{e}$ \\
& hcp & NM & $69.64(70.51)^{f}$ & $2.91(2.05)^{f}$ & 0.00 \\
$\mathrm{Ni}$ & bcc & FM & 74.23 & 1.88 & 0.52 \\
& fcc & FM & $73.43(73.65)^{c}$ & $2.00(1.86)^{c}$ & $0.60(0.61)^{c}$ \\
\hline \hline
\end{tabular}

Reference [12]

${ }^{b}$ Reference [16](Lattice constant extrapolated to $T=0 \mathrm{~K}$ )

" Reference [13] (Lattice constant extrapolated to $T=0 \mathrm{~K}$ )

${ }^{d}$ Reference [14] (Bulk modulus at $T_{R}$, magnetic moment at $T=0 \mathrm{~K}$ )

$e$ Reference [15] (Magnetic moment below $T_{N}$. )

$f$ W. Pepperhoff, unpublished

14\% [13]. From the results given in Fig. 2 we can conclude that the full potential LAPW method is correct for iron. It gives a good description of the structural and magnetic phases with the main results being in a good agreement with the experimental findings. Similar investigations have been done for nickel and manganese. Some of the most important results have already been mentioned (see Table 1). Nickel can be dealt with by using any band-structure theory. The same is correct for the structural states of manganese, but the description of the magnetic phases is improved by the full-potential method compared to methods without a self-interaction correction.

In the following part we will focus on the concentration dependent phase diagrams of the Fe-Mn and $\mathrm{Fe}-\mathrm{Ni}$ systems. From the total energies and magnetic moments for several stoichiometric ordered systems we extracted the concentration dependent structural phase diagram of these systems.

\subsection{Fe-Ni}

The theoretical and experimental phase diagram of the Fe-Ni alloy is given in Fig. 3. The most important result is connected with the energy difference $\Delta E_{\mathrm{fcc}-\mathrm{bcc}}$ between the fcc and bcc ground state, which can be related to the martensitic transition temperature. For $x \leq 70$ at $\%$ Fe the closepacked phase is always stable. Systems with larger iron concentrations prefer a bcc ground state. This is in excellent agreement with the experimental results, see left panel in Fig. 3. The absolute values of the transition temperatures can not be determined from our theoretical work. The behavior of the magnetic moments is comparable with experiment. $M_{\gamma}$ increases from $0.5 \mu_{B}$ for Ni to $2 \mu_{B}$ for 70 at\% Fe. For larger iron concentrations the magnetic moment of the $\gamma$ phase shrinks. That is connected with the onset of the martensitic phase transformation. In contrast to experiments we can simulate each phase over the whole concentration range and calculate $M_{\gamma}$ for fcc iron. But as can be seen in Fig. 2, this fcc phase is then higher in energy than bcc iron. On the Ni-rich side the magnetic moment compares qualitatively well with experiment. For iron it is known that bcc iron is stabilized by the ferromagnetic order (see section 3.1). The nonmagnetic and antiferromagnetic solutions have much higher energy. Without magnetism fcc would be always stable. We can conclude that there would be no martensitic $\alpha \rightarrow \gamma$ transformation without the magnetic order of iron.

Furthermore we have calculated the energy differences between ferromagnetic, antiferromagnetic and nonmagnetic fcc phases of Fe-Ni. $\Delta E_{\mathrm{FM}-\mathrm{NM}}^{\mathrm{fcc}}$ is a rough measure for the Curie temperature $T_{C}^{\gamma}$. 

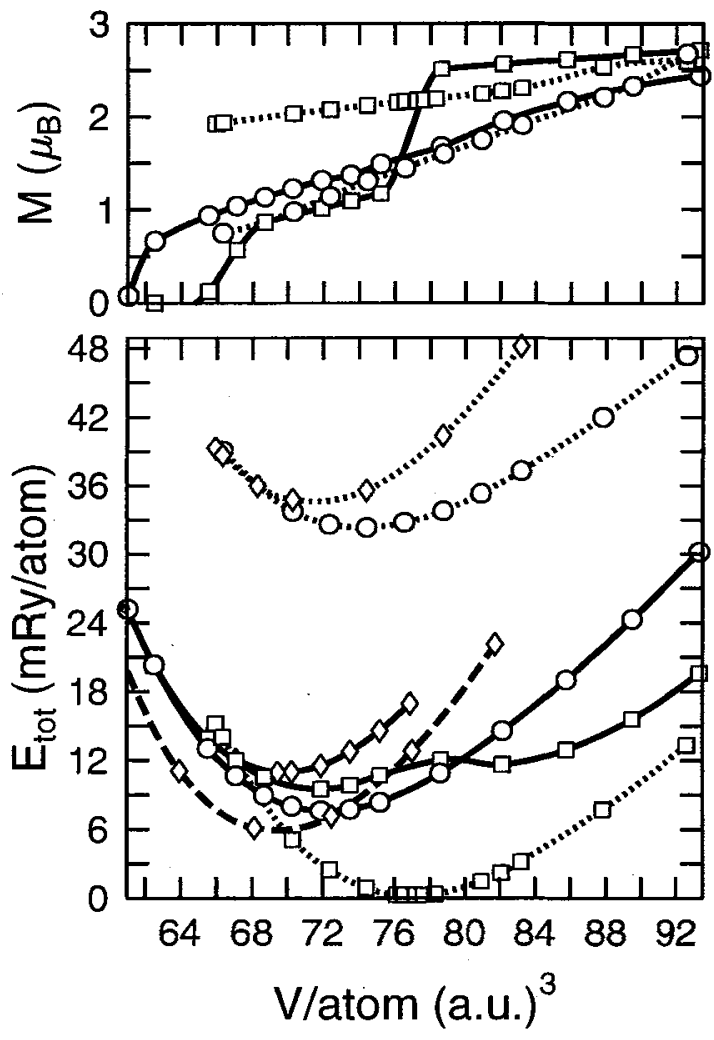

Figure 2: Total energies $E_{\text {tot }}$ (lower panel) and magnetic moments $M$ (upper panel) of iron obtained from FLAPW. calculations within the GGAII. Results are shown for the fcc and bcc crystal.structure and different magnetic ordering. Additionally the nonmagnetic hcp curve is presented (dashed line with diamonds). The solid curves corresponds to the fcc phase, and the dotted to the bcc phase. The diamonds indicate the nonmagnetic, the squares ferromagnetic and circles the antiferromagnetic order. For the ferromagnetic fcc phase exists a high-spin solution at $V_{0}^{\text {HS }}=81.15$ a.u. and a low-spin state at $V_{0}^{\mathrm{LS}}=71.69$ a.u. The sequence of the magnetic states of the fcc phase is important for the discussion of the Anti-Invar effect. This magnetovolume effect can be explained by thermal excitations from the AFM ground state to the highvolume HS FM state. The volume expansion is about $12.5 \%$ being close to the experimentally observed one.

The shape of the theoretical and experimental curves are identical as long as the fcc phase is the ground state. For larger iron concentrations it is not possible to measure $T_{C}^{\gamma}$. The Neél temperature, which can be described by $\Delta E_{\mathrm{FM}-\mathrm{AFM}}^{\mathrm{fcc}}$, shows also a qualitatively correct behavior. But in this case the calculated stability range is smaller. Only for iron concentrations larger than 87 at\% Fe the AFM state is stable, whereas in nature AFM ordering is possible at lower iron concentration. The last point to discuss is the concentration dependence of the Wigner-Seitz Radius $r_{W S}$. The maximum of the $r_{\mathrm{WS}}$ is found in the martensitic region about $80 \mathrm{at} \% \mathrm{Fe}$, whereas the maximum of the experimental curve occurs at 61 at\% $\mathrm{Fe}$ (Invar region). The shift to larger $r_{\text {ws }}$ may be caused by the stoichiometric order used in our calculations.

\subsection{Fe-Mn}

For the Fe-Mn system similar calculations have been done. The results are given in Fig. 4. In contrast to the $\mathrm{Fe}-\mathrm{Ni}$ compound, antiferromagnetism in $\mathrm{Fe}-\mathrm{Mn}$ is more important, because both $\mathrm{Fe}$ and $\mathrm{Mn}$, have an AFM fcc ground state. Again $M_{\gamma}$ follows the right chemical trend, but the absolute values obtained are slightly too large.

We found that for systems containing less than 80 at\% Fe the fcc phase is stable, larger iron concentrations leads to a bcc-like ground state. In nature only systems with more than 90 at\% $\mathrm{Fe}$ have a bcc ground state. For systems with Fe concentrations between 80 and 90 at $\%$ a hcp $\rightarrow$ fcc transformation plays an important role in the Fe-Mn system. 

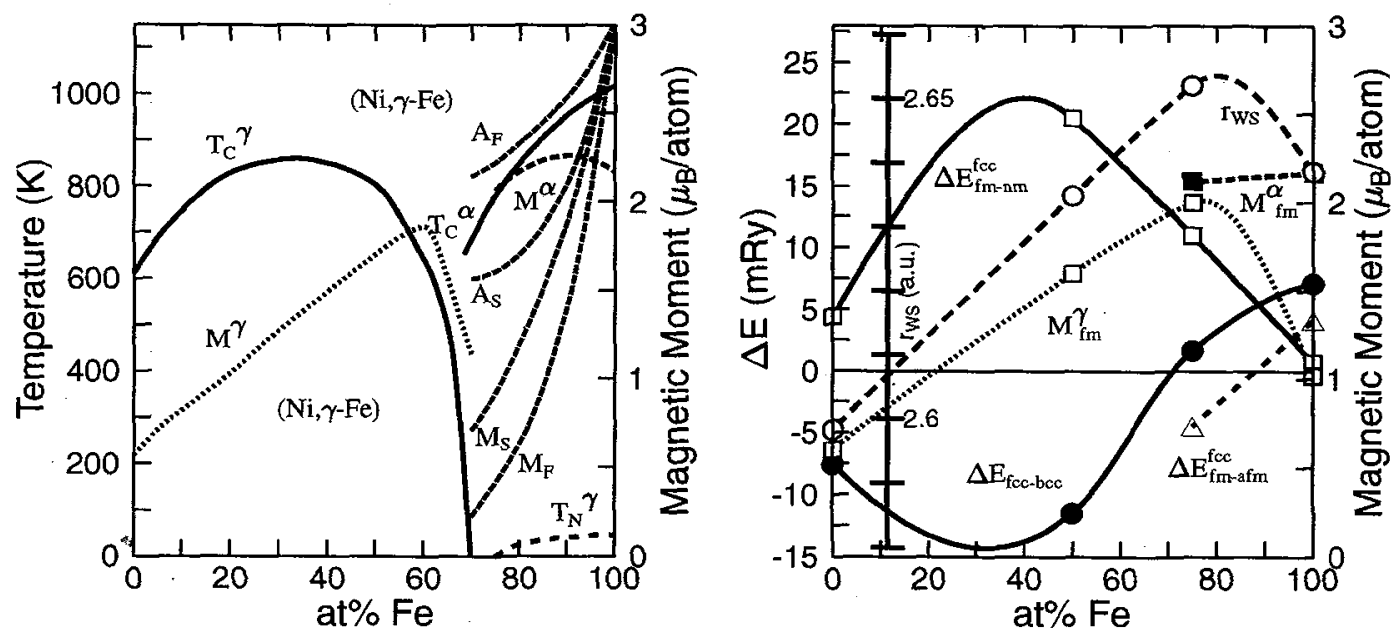

Figure 3: Experimental (left) and theoretical (right) phase diagram of Fe-Ni. The labels $\gamma, \alpha$ refer to the fcc, bcc structures. Temperatures in the left figure are Curie $\left(T_{C}\right)$ and Neel $\left(T_{N}\right)$ temperatures. Austenite/martensite start and final temperatures are labeled by $A_{F}, A_{S}, M_{S}, M_{F} . M^{\gamma}\left(M^{\alpha}\right)$ denotes the saturation magnetization of the fcc (bcc) phase. The theoretical energy differences can be compared with the temperatures in the left panel. $\Delta E_{\mathrm{NM}-\mathrm{FM}}^{\mathrm{fcc}}$ is correlated to $T_{C}^{\gamma}$ and $\Delta E_{\mathrm{FM}-\mathrm{AFM}}^{\mathrm{fcc}}$ simulates $T_{N}^{\gamma} . \Delta E_{\mathrm{fcc}-\mathrm{bcc}}$ determines the stability range of an fcc ground state. The inset scale in the right panel belongs to the Wigner-Seitz radius $r_{W s}$ (dashed line with open circles). For details see text.
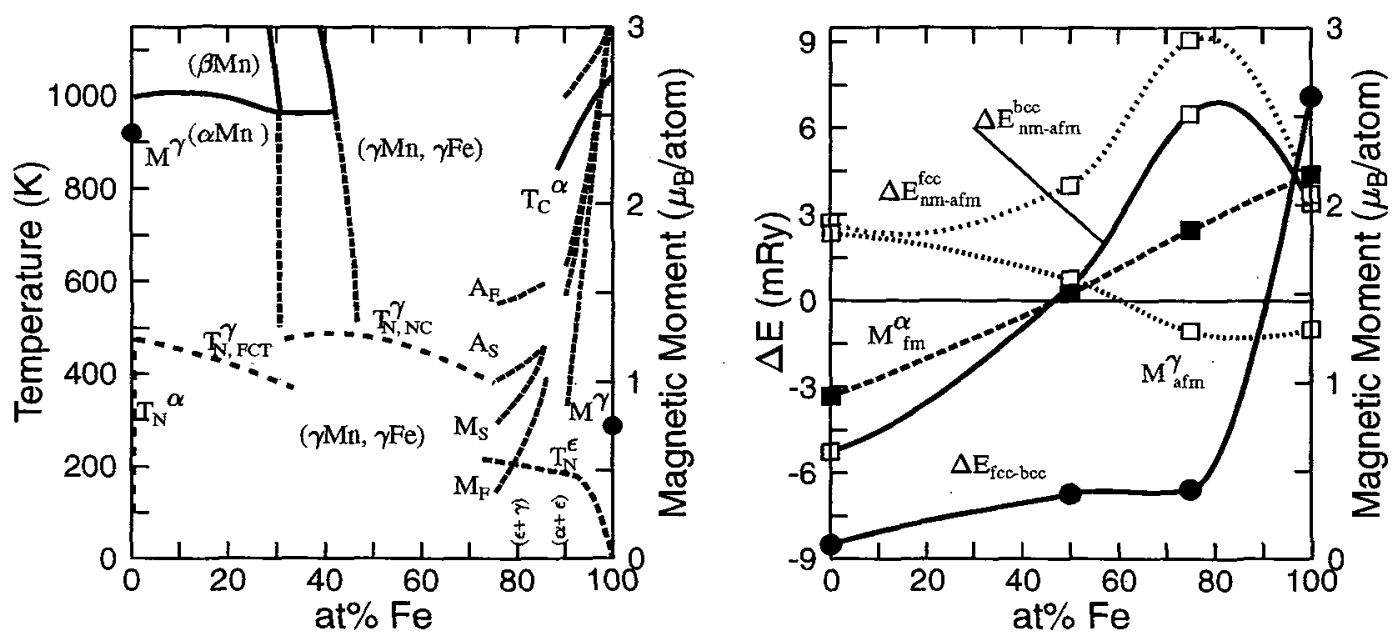

Figure 4: Experimental (left) and theoretical (right) phase diagram of Fe-Mn. The labels $\gamma, \alpha$ refer to the fcc, bcc structures. The temperatures on the left figure are Curie $\left(T_{C}\right)$ and Neél $\left(T_{N}\right)$ temperatures. Austenite/martensite start and final temperatures are labeled by $A_{F}, A_{S}, M_{S}, M_{F} . M^{\gamma}$ denotes the saturation magnetization of fcc Mn and fcc Fe. The theoretical energy differences can be compared to the temperatures in the left panel. $\triangle E_{N M-A F M}^{\mathrm{fcc}}$ is correlated to $T_{N}^{\gamma}$. $\Delta E_{\mathrm{fcc}-\mathrm{bcc}}$ determines the stability range of an fcc ground state. For more information see text. 
This can not be seen in the theoretical phase diagram, where hcp.or mixed phases have not been considered. Additionally we have examined total energy differences of the magnetic phases. We found a weak concentration dependence of the Neél temperature being correlated to $\Delta E_{\mathrm{NM}-\mathrm{AFM}}^{\mathrm{fcc}}$. This agrees with the experimental observations (see Fig. 4).

\section{CONCLUSIONS}

In this paper we have presented theoretical phase diagrams for two transition metal alloys. Though only four stoichiometric ordered systems have been calculated, we can draw some interesting conclusions from the results mentioned above. Characteristic features of iron still exist in $\mathrm{Fe}-\mathrm{Ni}$ alloys in the martensitic region. In systems containing more than 70 at\% iron the structural as well as the magnetic properties are dominated by FM bcc iron. However, the Fe-Mn system behaves differently. First, this system is nearly AFM for all concentrations, because antiferromagnetism is favored by Mn. On the other hand antiferromagnetism prefers close packed structures. From our calculations we may conclude that the bcc phase of Fe- $\mathrm{Mn}$ is already destabilized for Fe concentrations less than $90 \mathrm{at} \%$, which cloes qualitatively agree with experiments. The reason that bcc Fe- $\mathrm{Mn}$ exist only in a small concentration region, is the AFM character of $\mathrm{Mn}$, because bcc is only stabilized through FM (see section 3.2.).

At least one should keep in mind that only cubic phases are discussed though these alloys show also $c x \rightarrow \epsilon$ transitions. This will be discussed in a forthcoming paper.

\section{Acknowledgments}

One of us (H.C. H.) would like to thank M. Acet and W. Pepperhoff for fruitful discussions.

\section{References}

[1] Wassermann E.F., Ferromagnetic Materials (North-Holland, Amsterdam, 1990) , pp. 240.

[2] Moruzzi V.L., Marcus P.M., Schwarz K., and Mohn P., Phys. Rev. B 34 (1986) 1784-1791.

[3] Asada T. and Terakura K., Phys. Rev. B 46 (1992) 13599-13602.

[4] Wang C.S., Klein B.M., and Krakauer H., Phys. Rev. Lett. 54 (1985) 1852-1855.

[5] Singh D.J., Planewaves, Pseudopotentials and the LAPW method (Kluwer Acad., Boston, 1994).

[6] Blaha P., Schwarz K., Dufek P., and Augustyn R., WIEN95, TU of Vienna 1995. (Improved and updated Unix version of the original copyrighted WIEN-code, published by Blaha P., Schwarz K., Sorantin P., and Trickey S.B., in Comput. Phys. Commun. 59 (1990) 399).

[7] Perdew J.P., and Wang Y., Phys Rev. B 45 (1992) 13244-13249.

[8] Murnaghan F.D., Proc. Nat. Acad. Sci. USA 30 (1944) 244-247.

[9] Herper H.C., Hoffmann E., and Entel P., "Ab initio investigations of phonon anomalies and martensitic transformations", IAC1, Athens 16-21 June 1996, Plenum, in press.

[10] Herper H.C., Hoffmann E. and Entel P., in preparation

[11] Donohue J., The structures of the elements (Robert Krieger Publ., Florida, 1982). pp. 198-200.

[12] Tebble R.S., Craik D.J., Magnetic Materials (Wiley \& Sons, London,1969), p. 62.

[13] Acet M., Zähres H., and Wassermann E.F., Phys. Rev. B 49 (1994) 6012.

[14] Kittel C., Introduction to Solid State Physics (Wiley \& Sons, New York, 1971, 4th ed.) pp. $143+536$.

[15] Abrahams S.C., Guttman L., and Kasper J.S.,Phys. Rev 127 (1962) 2052-2055.

[16] Acet M., Zähres H., Stamm W., Wassermann E.F., and Pepperhoff W., Physica B 161 (1989) $67-71$. 